\title{
ON EXTENSIONS OF COVARIANTLY FINITE SUBCATEGORIES
}

\author{
XIAO-WU CHEN
}

\author{
Department of Mathematics \\ University of Science and Technology of China \\ Hefei 230026, P. R. China
}

\begin{abstract}
In [6], Gentle and Todorov proved that in an abelian category with enough projective objects, the extension subcategory of two covariantly finite subcategories is still covariantly finite. We give an counterexample to show that Gentle-Todorov's theorem may fail in arbitrary abelian categories; we also prove that a triangulated version of Gentle-Todorov's theorem holds; we make applications of Gentle-Todorov's theorem to obtain short proofs to a classical result by Ringel and a recent result by Krause and Solberg.
\end{abstract}

\section{Main Theorems}

Let $\mathcal{C}$ be an additive category. By a subcategory $\mathcal{X}$ of $\mathcal{C}$ we always mean a full additive subcategory. Let $\mathcal{X}$ be a subcategory of $\mathcal{C}$ and let $M \in \mathcal{C}$. A morphism $x_{M}: M \longrightarrow X_{M}$ is called a left $\mathcal{X}$-approximation of $M$ if $X_{M} \in \mathcal{X}$ and every morphism from $M$ to an object in $\mathcal{X}$ factors through $x_{M}$. The subcategory $\mathcal{X}$ is said to be covariantly finite in $\mathcal{C}$, if every object in $\mathcal{C}$ has a left $\mathcal{X}$-approximation. The notions of left $\mathcal{X}$-approximation and covariantly finite are also known as $\mathcal{X}$-preenvelop and preenveloping, respectively. For details, see [3, 4] and [5].

To state our main result, let $\mathcal{C}$ be an abelian category. Let $\mathcal{X}$ and $\mathcal{Y}$ be its subcategories. Set $\mathcal{X} * \mathcal{Y}$ to be the subcategory consisting of objects $Z$ such that there is a short exact sequence $0 \longrightarrow X \longrightarrow Z \longrightarrow Y \longrightarrow 0$ with $X \in \mathcal{X}$ and $Y \in \mathcal{Y}$, and it is called the extension subcategory of $\mathcal{Y}$ by $\mathcal{X}$. Note that the operation "*" on subcategories is associative. Recall that an abelian category $\mathcal{C}$ has enough projective objects, if for each object $M$ there is an epimorphism $P \longrightarrow M$ with $P$ projective.

The following result is due to Gentle and Todorov [6], which extends the corresponding results in artin algebras and coherent rings, obtained by Sikko and Smalø (see [11, Theorem 2.6] and [12]).

Theorem 1.1. (Gentle-Todorov [6, Theorem 1.1, ii)]) Let $\mathcal{C}$ be an abelian category with enough projective objects. Assume that both $\mathcal{X}$ and $\mathcal{Y}$ are covariantly finite subcategories in $\mathcal{C}$. Then the extension subcategory $\mathcal{X} * \mathcal{Y}$ is covariantly finite.

This project was supported by China Postdoctoral Science Foundation No. 20070420125, and was also partially supported by the National Natural Science Foundation of China (Grant No.s 10501041 and 10601052). The author also gratefully acknowledges the support of K. C. Wong Education Foundation, Hong Kong.

E-mail: xwchen@mail.ustc.edu.cn. 
Proof. The proof is also due to Gentle and Todorov, and here we just include it for an inspiration of the proof of Theorem 1.4. Note that the argument here resembles the one in the proof of [8, Lemma 1.3].

Assume that $M \in \mathcal{C}$ is an arbitrary object. Take its left $\mathcal{Y}$-approximation $y_{M}$ : $M \longrightarrow Y_{M}$ with $Y_{M} \in \mathcal{Y}$. By assumption the category $\mathcal{C}$ has enough projective objects, we may take an epimorphism $\pi_{M}: P \longrightarrow Y_{M}$ with $P$ projective. Consider the short exact sequence $0 \longrightarrow K \longrightarrow M \oplus P \stackrel{\left(y_{M}, \pi_{M}\right)}{\longrightarrow} Y_{M} \longrightarrow 0$. Take a left $\mathcal{X}$ approximation $x_{K}: K \longrightarrow X_{K}$ of $K$. We form a pushout and then get the following commutative exact diagram

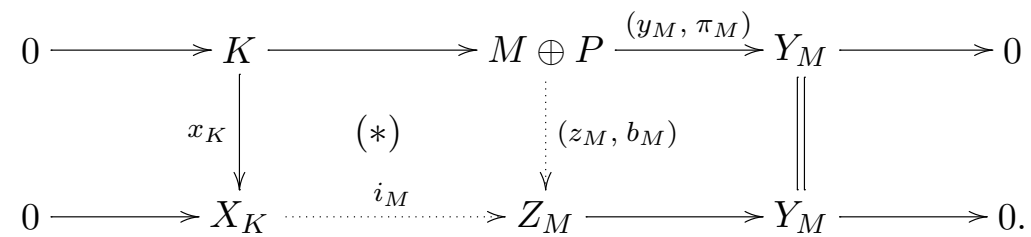

Note that $Z_{M} \in \mathcal{X} * \mathcal{Y}$. We claim that the morphism $z_{M}: M \longrightarrow Z_{M}$ is a left $\mathcal{X} * \mathcal{Y}$-approximation of $M$. Then we are done.

To see this, assume that we are given a morphism $f: M \longrightarrow Z$, and $Z \in \mathcal{X} * \mathcal{Y}$, that is, there is an exact sequence $0 \longrightarrow X \stackrel{i}{\longrightarrow} Z \stackrel{\pi}{\longrightarrow} Y \longrightarrow 0$ with $X \in \mathcal{X}$ and $Y \in \mathcal{Y}$. Since $y_{M}$ is a left $\mathcal{Y}$-approximation, then the composite morphism $\pi \circ f$ factors through $y_{M}$, say there is a morphism $c: Y_{M} \longrightarrow Y$ such that $\pi \circ f=c \circ y_{M}$. Consider the composite morphism $P \stackrel{\pi_{M}}{\longrightarrow} Y_{M} \stackrel{c}{\longrightarrow} Y$ and the epimorphism $\pi: Z \longrightarrow Y$. Since $P$ is projective, we have a morphism $b: P \longrightarrow Z$ such that $\pi \circ b=c \circ \pi_{M}$. Hence we have the following commutative exact diagram.

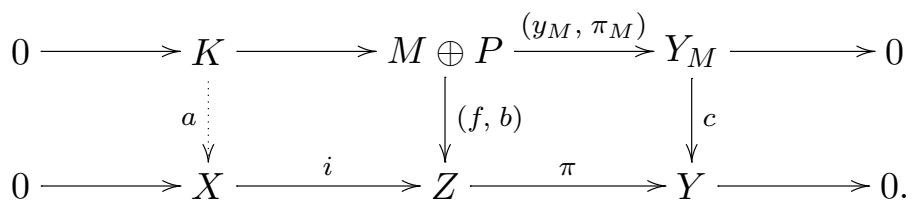

Since $x_{K}$ is a left $\mathcal{X}$-approximation, the morphism $a$ factors through $x_{K}$, say we have $a=a^{\prime} \circ x_{K}$ with $a^{\prime}: X_{K} \longrightarrow X$. Then by the universal mapping property of the pushout square $(*)$, there is a unique morphism $h: Z_{M} \longrightarrow Z$ such that

$$
h \circ i_{M}=i \circ a^{\prime}, \quad \text { and } h \circ\left(z_{M}, b_{M}\right)=(f, b) .
$$

In particular, the morphism $f$ factors through $z_{M}$, as required.

Remark 1.2. The same proof yields another version of Gentle-Todorov's theorem ([6. Theorem 1.1, i)]): Let $\mathcal{C}$ be an abelian category, $\mathcal{X}$ and $\mathcal{Y}$ covariantly finite subcategories in $\mathcal{C}$. Assume further that $\mathcal{Y}$ is closed under subobjects. Then the extension subcategory $\mathcal{X} * \mathcal{Y}$ is covariantly finite. (In the proof of this case, $y_{M}$ could be assumed to be epic, and then we just take $P=0$.)

Example 1.3. We remark that Gentle-Todorov's theorem may fail if the abelian category has not enough projective objects. To give an example, let $k$ be a field, and 
let $Q$ be the following quiver

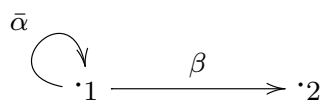

where $\bar{\alpha}=\left\{\alpha_{i}\right\}_{i \geq 1}$ is a family of arrows indexed by positive numbers. Recall that a representation of $Q$, denoted by $V=\left(V_{1}, V_{2} ; V_{\bar{\alpha}}, V_{\beta}\right)$, is given by the following data: two $k$-spaces $V_{1}$ and $V_{2}$ attached to the vertexes, and $V_{\bar{\alpha}}=\left(V_{\alpha_{i}}\right)_{i \geq 1}, V_{\alpha_{i}}$ : $V_{1} \longrightarrow V_{1}$ and $V_{\beta}: V_{1} \longrightarrow V_{2} k$-linear maps attached to the arrows. A morphism of representations, denoted by $f=\left(f_{1}, f_{2}\right): V \longrightarrow V^{\prime}$, consists of two linear maps $f_{i}: V_{i} \longrightarrow V_{i}^{\prime}, i=1,2$, which are compatible with the linear maps attached to the arrows. Denote by $\mathcal{C}$ the category of representations $V=\left(V_{1}, V_{2} ; V_{\bar{\alpha}}, V_{\beta}\right)$ of $Q$ such that $\operatorname{dim}_{k} V=\operatorname{dim}_{k} V_{1}+\operatorname{dim}_{k} V_{2}<\infty$ and $V_{\alpha_{i}}$ are zero for all but finitely many $i$ 's. Then $\mathcal{C}$ is an abelian category with finite-dimensional Hom spaces.

Denote by $S_{i}$ the one-dimensional representation of $Q$ seated on the vertex $i$ with zero maps attached to all the arrows, $i=1,2$. Consider the two-dimensional representation $M=\left(M_{1}=k, M_{2}=k ; M_{\bar{\alpha}}=0, M_{\beta}=1\right)$. Denote by $\mathcal{X}$ (resp. $\left.\mathcal{Y}\right)$ the subcategory consisting of direct sums of copies of $S_{1}$ (resp. $M$ ). Then both $\mathcal{X}$ and $\mathcal{Y}$ are covariantly finite in $\mathcal{C}$. However we claim that $\mathcal{Z}=\mathcal{X} * \mathcal{Y}$ is not covariantly finite.

In fact, the representation $S_{2}$ does not have a left $\mathcal{Z}$-approximation. Otherwise, assume that $\phi: S_{2} \longrightarrow V$ is a left $\mathcal{Z}$-approximation. Take $i_{0}>>1$ such that $V_{\alpha_{i_{0}}}$ is a zero map. Consider the following three-dimensional representation

$$
W=\left(W_{1}=\left(\begin{array}{l}
k \\
k
\end{array}\right), W_{2}=k ; W_{\alpha_{i_{0}}}=\left(\begin{array}{ll}
0 & 0 \\
1 & 0
\end{array}\right), W_{\alpha_{i}}=0 \text { for } i \neq i_{0}, W_{\beta}=(1,0)\right) .
$$

We have a non-split exact sequence of representations $0 \longrightarrow S_{1} \longrightarrow W \longrightarrow M \longrightarrow 0$. In particular, $W \in \mathcal{Z}$. Note that $\operatorname{Hom}_{\mathcal{C}}\left(S_{2}, W\right) \simeq k$. Hence there is a morphism $f=\left(f_{1}, f_{2}\right): V \longrightarrow W$ such that $f \circ \phi \neq 0$. However this is not possible. Note that $W_{\alpha_{i_{0}}}\left(f_{1}\left(V_{1}\right)\right)=f_{1}\left(V_{\alpha_{i_{0}}}\left(V_{1}\right)\right)=0$ by the choice of $i_{0}$, and that Ker $W_{\alpha_{i_{0}}}=$ Ker $W_{\beta}$, we obtain that $W_{\beta}\left(f_{1}\left(V_{1}\right)\right)=0$. Note that both the representations $S_{1}$ and $M$ satisfy that the map attached to the arrow $\beta$ is surjective, and then by Snake Lemma we infer that every representation in $\mathcal{Z}$ has this property, in particular, the representation $V$ has this property, that is, $V_{2}=V_{\beta}\left(V_{1}\right)$. Hence we have $0=$ $W_{\beta}\left(f_{1}\left(V_{1}\right)\right)=f_{2}\left(V_{\beta}\left(V_{1}\right)\right)=f_{2}\left(V_{2}\right)$, and thus we deduce that $f_{2}=0$. This will force that the composite $S_{2} \stackrel{\phi}{\longrightarrow} V \stackrel{f}{\longrightarrow} W$ is zero.

We also have a triangulated version of Gentle-Todorov's theorem. Let $\mathcal{C}$ be a triangulated category with the translation functor denoted by [1]. For triangulated categories, we refer to [13, 7]. Let $\mathcal{X}, \mathcal{Y}$ be its subcategories. Set $\mathcal{X} * \mathcal{Y}$ to be the extension subcategory, that is, the subcategory consisting of objects $Z$ such that there is a triangle $X \longrightarrow Z \longrightarrow Y \longrightarrow X[1]$ with $X \in \mathcal{X}$ and $Y \in \mathcal{Y}$. Again this operation "*" on subcategories is associative by the octahedral axiom (TR4). Then we have the following result.

Theorem 1.4. Let $\mathcal{C}$ be a triangulated category. Assume that both $\mathcal{X}$ and $\mathcal{Y}$ are covariantly finite subcategories in $\mathcal{C}$. Then the extension subcategory $\mathcal{X} * \mathcal{Y}$ is covariantly finite. 
Proof. As we noted above, the proof here is a triangulated version of the proof of Gentle-Todorov's theorem. Assume that $M \in \mathcal{C}$ is an arbitrary object. Take its left $\mathcal{Y}$-approximation $y_{M}: M \longrightarrow Y_{M}$ with $Y_{M} \in \mathcal{Y}$. Form a triangle $K \stackrel{k}{\longrightarrow} M \stackrel{y_{M}}{\longrightarrow}$ $Y_{M} \longrightarrow K[1]$. Take a left $\mathcal{X}$-approximation $x_{K}: K \longrightarrow X_{K}$ of $K$.

Recall from [7, Appendix] that the octahedral axiom (TR4) is equivalent to the axioms (TR4') and (TR4"). Hence we have a commutative diagram of triangles

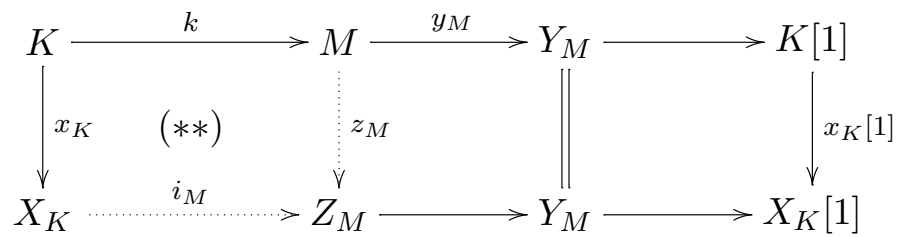

where the square $(* *)$ is a homotopy cartesian square, that is, there is a triangle

$$
K \stackrel{\left(\begin{array}{c}
k \\
x_{K}
\end{array}\right)}{\longrightarrow} M \oplus X_{K} \stackrel{\left(z_{M},-i_{M}\right)}{\longrightarrow} Z_{M} \rightarrow K[1] .
$$

Note that $Z_{M} \in \mathcal{X} * \mathcal{Y}$. We claim that the morphism $z_{M}: M \longrightarrow Z_{M}$ is a left $\mathcal{X} * \mathcal{Y}$-approximation of $M$. Then we are done.

To see this, assume that we are given a morphism $f: M \longrightarrow Z$, and $Z \in \mathcal{X} * \mathcal{Y}$, that is, there is a triangle $X \stackrel{i}{\longrightarrow} Z \stackrel{\pi}{\longrightarrow} Y \longrightarrow X[1]$ with $X \in \mathcal{X}$ and $Y \in \mathcal{Y}$. Since $y_{M}$ is a left $\mathcal{Y}$-approximation, then the composite morphism $\pi \circ f$ factors through $y_{M}$, say there is a morphism $c: Y_{M} \longrightarrow Y$ such that $\pi \circ f=c \circ y_{M}$. Hence by the axiom (TR3), we have a commutative diagram

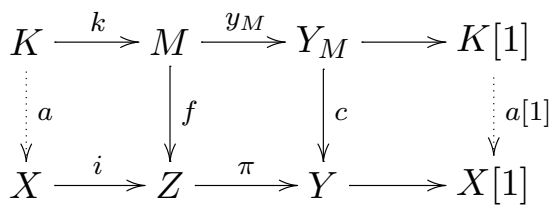

Since $x_{K}$ is a left $\mathcal{X}$-approximation, the morphism $a$ factors through $x_{K}$, say we have $a=a^{\prime} \circ x_{K}$ with $a^{\prime}: X_{K} \longrightarrow X$. Hence $\left(i \circ a^{\prime}\right) \circ x_{K}=f \circ k$, and thus $\left(f,-i \circ a^{\prime}\right) \circ\left(\begin{array}{c}k \\ x_{K}\end{array}\right)=0$. Applying the cohomological functor $\operatorname{Hom}_{\mathcal{C}}(-, Z)$ to the triangle (1.1), we deduce that there is a morphism $h: Z_{M} \longrightarrow Z$ such that

$$
h \circ\left(z_{M},-i_{M}\right)=\left(f,-i \circ a^{\prime}\right) .
$$

In particular, the morphism $f$ factors through $z_{M}$, as required.

\section{Applications of Gentle-Todorov's Theorem}

In this section, we apply Gentle-Todorov's theorem to the representation theory of artin algebras. We obtain short proofs of a classical result by Ringel and a recent result by Krause and Solberg.

Let $A$ be an artin algebra, $A$-mod the category of finitely generated left $A$-modules. Dual to the notions of left approximations and covariantly finite subcategories, we have the notions of right approximations and contravariantly finite subcategories. A subcategory is called functorially finite, it is both covariantly finite and contravariantly finite. All these properties are called homologically finiteness properties. 
We need more notation. Let $\mathcal{X} \subseteq A$-mod be a subcategory. Set add $\mathcal{X}$ to be its additive closure, that is, the subcategory consisting of direct summands of modules in $\mathcal{X}$. Note that the subcategory $\mathcal{X}$ has these homological finiteness properties if and only if add $\mathcal{X}$ does. Let $r \geq 1$ and $\mathcal{X}$ a subcategory of $A$-mod. Set $\mathcal{F}_{r}(\mathcal{X})=$ $\mathcal{X} * \mathcal{X} * \cdots * \mathcal{X}$ (with $r$-copies of $\mathcal{X}$ ). Hence a module $M$ lies in $\mathcal{F}_{r}(\mathcal{X})$ if and only if $M$ has a filtration of submodules $0=M_{0} \subseteq M_{1} \subseteq M_{2} \subseteq \cdots \subseteq M_{r}=M$ with each factors $M_{i} / M_{i-1}$ in $\mathcal{X}$.

Note that the abelian category $A$-mod has enough projective and enough injective objects, and thus Gentle-Todorov's theorem and its dual (on contravariantly finite subcategories) hold. Thus the following result is immediate.

Corollary 2.1. ([11, Corollary 2.8]) Let $r \geq 1$ and $\mathcal{X}$ a subcategory of A-mod. Assume that $\mathcal{X}$ is covariantly finite (resp. contravariantly finite, functorially finite). Then the subcategories $\mathcal{F}_{r}(\mathcal{X})$ and add $\mathcal{F}_{r}(\mathcal{X})$ are covariantly finite (resp. contravariantly finite, functorially finite).

Recall that a subcategory $\mathcal{X}$ in $A$-mod is said to be a finite subcategory provided that there is a finite set of modules $X_{1}, X_{2}, \cdots, X_{r}$ in $\mathcal{X}$ such that each module in $\mathcal{X}$ is a direct summand of direct sums of copies of $X_{i}$ 's. Finite subcategories are functorially finite ([3, Proposition 4.2]). Let $r, n \geq 1$ and $\mathcal{S}=\left\{X_{1}, X_{2}, \cdots, X_{n}\right\}$ a finite set of modules, denote by $\mathcal{S}^{\oplus}$ the subcategory consisting of direct sums of copies of modules in $\mathcal{S}$; for each $n \geq 1$, set $\mathcal{F}_{r}(\mathcal{S})=\mathcal{F}_{r}\left(\mathcal{S}^{\oplus}\right)$. Note that $\mathcal{S}^{\oplus}$ is a finite subcategory and thus a functorially finite subcategory. The following is a direct consequence of Corollary 2.1.

Corollary 2.2. Let $r, n \geq 1$, and $\mathcal{S}=\left\{X_{1}, X_{2}, \cdots X_{n}\right\}$ a finite set of $A$-modules. Then the subcategories $\mathcal{F}_{r}(\mathcal{S})$ and add $\mathcal{F}_{r}(\mathcal{S})$ are functorially finite.

Let $n \geq 1$ and $\mathcal{S}$ be as above. Ringel introduces in 9 the subcategory $\mathcal{F}(\mathcal{S})$ to be the subcategory consisting of modules $M$ with a filtration of submodules $0=M_{0} \subseteq$ $M_{1} \subseteq M_{2} \subseteq \cdots \subseteq M_{r}=M$ with $r \geq 1$ and each factor $M_{i} / M_{i-1}$ belonging to $\mathcal{S}$. One observes that $\mathcal{F}(\mathcal{S})=\bigcup_{r \geq 1} \mathcal{F}_{r}(\mathcal{S})$. Then we obtain the following classical result of Ringel with a short proof.

Corollary 2.3. (Ringel, [9, Theorem 1] and [10]) Let $n \geq 1$ and $\mathcal{S}=\left\{X_{1}, X_{2}, \cdots, X_{n}\right\}$ a finite set of $A$-modules. Assume that $\operatorname{Ext}_{A}^{1}\left(X_{i}, X_{j}\right)=0$ for $i \leq j$. Then the subcategory $\mathcal{F}(\mathcal{S})$ is functorially finite.

Proof. First note the following factors-exchanging operation: let $M$ be a module with a filtration of submodules

$$
0=M_{0} \subseteq M_{1} \subseteq \cdots \subseteq M_{i-1} \subseteq M_{i} \subseteq M_{i+1} \subseteq \cdots \subseteq M_{r}=M,
$$

and we assume that $\operatorname{Ext}_{A}^{1}\left(M_{i+1} / M_{i}, M_{i} / M_{i-1}\right)=0$ for some $i$, then $M$ has a new filtration of submodules

$$
0=M_{0} \subseteq M_{1} \subseteq \cdots \subseteq M_{i-1} \subseteq M_{i}^{\prime} \subseteq M_{i+1} \subseteq \cdots \subseteq M_{r}=M
$$

exchanging the factors at $i$, that is, $M_{i}^{\prime} / M_{i-1} \simeq M_{i+1} / M_{i}$ and $M_{i+1} / M_{i}^{\prime} \simeq M_{i} / M_{i-1}$.

We claim that $\mathcal{F}(\mathcal{S})=\mathcal{F}_{n}(\mathcal{S})$. Then by Corollary 2.2 we are done. Let $M \in \mathcal{F}(\mathcal{S})$. By iterating the factors-exachanging operations, we may assume that the module $M$ 
has a filtration $0=M_{0} \subseteq M_{1} \subseteq M_{2} \subseteq \cdots \subseteq M_{r}=M$ such that there is a sequence of numbers $1 \leq r_{1} \leq r_{2} \leq \cdots \leq r_{n}=r$ satisfying that the factors $M_{j} / M_{j-1} \simeq X_{i}$ for $r_{i-1}+1 \leq j \leq r_{i}$ (where $r_{0}=0$ ). Because of $\operatorname{Ext}_{A}^{1}\left(X_{i}, X_{i}\right)=0$, we deduce that $M_{r_{i}} / M_{r_{i-1}+1}$ is a direct sum of copies of $X_{i}$ for each $1 \leq i \leq n$. Therefore $M \in \mathcal{F}_{n}(\mathcal{S})$, as required.

We will give a short proof to a surprising result recently obtained by Krause and Solberg [8]. Note that their proof uses cotorsion pairs on the category of infinite length modules essentially, while our proof uses only finite length modules. Recall that a subcategory $\mathcal{X}$ of $A$-mod is resolving if it contains all projective modules and it is closed under extensions, kernels of epimorphims and direct summands ([1, p.99]).

Corollary 2.4. (Krause-Solberg, [8, Corollary 0.3]) A resolving contravarianly finite subcategory of $A$-mod is covariantly finite, and thus functorially finite,

Proof. Let $\mathcal{X} \subseteq A$-mod be a resolving contravariantly finite subcategory. Assume that $\left\{S_{1}, S_{2}, \cdots, S_{n}\right\}$ is the complete set of pairwise nonisomorphic simple $A$-modules, and take, for each $i$, the minimal right $\mathcal{X}$-approximation $X_{i} \longrightarrow S_{i}$. Set $\mathcal{S}=\left\{X_{1}, X_{2}, \cdots, X_{n}\right\}$. Denote by $J$ the Jacobson idea of $A$, and assume that $J^{r}=0$ for some $r \geq 1$. Thus every modules $M$ has a filtration $0=M_{0} \subseteq M_{1} \subseteq \cdots \subseteq M_{r}=$ $M$ with semisimple factors. Hence by [2, Propostion 3.8], or more precisely by the proof [2, Proposition 3.7 and 3.8], we have that $\mathcal{X}=$ add $\mathcal{F}_{n}(\mathcal{S})$. By Corollary 2.2 the subcategory $\mathcal{X}$ is functorially finite.

Let us end with an example of functorially finite subcategories.

Example 2.5. Let $A$ be an artin algebra and $I$ a two-sided ideal of $A$ such that the quotient algebra $A / I$ is of representation finite type, that is, there are only finitely many isoclasses of (finitely generated) indecomposable $A / I$-modules. For example, the Jacobson ideal $J$ satisfies this condition. Let $r \geq 1$, and let $\mathcal{X}_{r}$ be the subcategory of $A$-mod consisting of modules annihilated by $I^{r}$. We claim that the subcategory $\mathcal{X}_{r}$ is functorially finite in $A$-mod.

To see this, first note that the subcategory $\mathcal{X}_{1}$ could be identified with $A / I$-mod, and hence by assumption $\mathcal{X}_{1}$ is a finite subcategory, and thus functorially finite in $A$-mod. Then it is a pleasant exercise to check that $\mathcal{X}_{r}=\mathcal{F}_{r}\left(\mathcal{X}_{1}\right)$. By Corollary 2.1 we deduce that the subcategory $\mathcal{X}_{r}$ is functorially finite.

Acknowledgement. The author would like to thank Prof. Henning Krause very much, who half a year ago asked him to give a direct proof to their surprising result [8, Corollary 0.3]. Thanks also go to Dr. Yu Ye for pointing out the references [11, 12] and special thanks go to Prof. Apostolos Beligiannis who kindly pointed out to the author that Theorem 1.1 and the proof were originally due to Gentle and Todorov.

\section{REFERENCES}

[1] M. Aulsnader And M. Bridger, Stable Module Theory, Mem. Amer. Math. Soc. 94, Amer. Math. Soc., Providence, R. I., 1969.

[2] M. Auslander and I. Reiten, Stable equivalence of dualizing R-varieties, Adv. Math. 12 (1974), 306-366.

[3] M. Auslander And S.O. Smalø, Preprojective modules over artin algebras, J. Algebra 66 (1980), 61-122. 
[4] M. Auslander And S.O. Smalø, Almost split sequences in subcategories, J. Algebra 69 (1981), 426-454.

[5] E.E. Enochs, Injective and flat covers, envelopes and resolvents, Israel J. Math. 39 (1981), 189-209.

[6] R. Gentle And G. Todorov, Extensions, kernels and cokernels of homologically finite subcategories, in: Representation theory of algebras (Cocoyoc, 1994), 227-235, CMS Conf. Proc. 18, Amer. Math. Soc., Providence, RI, 1996.

[7] H. Krause, Derived categories, resolutions, and Brown representability, in: Interactions between homotopy theory and algebra, 101-139, Contemp. Math. 436, Amer. Math. Soc., Providence, RI, 2007.

[8] H. Krause and Ø. Solberg, Applications of cotorsion pairs, J. London Math. Soc. (2) 68 (2003), 631-650.

[9] C.M. RingeL, The category of mofules with good filtrations over a quasi-hereditary algebras has almost split sequences, Math. Z. 208 (1991), 209-223.

[10] C.M. Ringel, On contravariantly finite subcategories, Proceedings of the Sixth Inter. Confer. on Representations of Algebras (Ottawa, ON, 1992), 5 pp., Carleton-Ottawa Math. Lecture Note Ser. 14, Carleton Univ., Ottawa, ON, 1992.

[11] S.A. Sikko And S.O. Smalø, Extensions of homological finite subcategories, Arch. Math. 60 (1993), 517-526.

[12] S.A. Sikko And S.O. Smalø, Coherent rings and homologically finite subcategories, Math. Scand. 77 (2)(1995), 175-183.

[13] J.L. Verdier, Catégories dérivées, etat 0, Springer Lecture Notes 569 (1977), 262-311. 